\title{
An Incidental Finding of Unroofed Coronary Sinus During Angiography Confirmed By 3D Echocardiography; A Case Report
}

\author{
Afsoon Fazlinezhad ${ }^{1}$, Faeze Keihanian ${ }^{1}$, Mostafa Ahmadi $^{1}$, and Mohammad Tayyebi ${ }^{1}$ \\ ${ }^{1}$ Mashhad University of Medical Sciences
}

November 1, 2021

\begin{abstract}
Unroofed Coronary Sinus (UCS) is an uncommon congenital heart anomaly and the most unusual type of Atrial Septal Defect.This report presents a-71-year-old female with palpitation and dyspnea following an emotional stress, who was referred for ablation of typical atrial flutter.Finally diagnosed with a partially UCS causing a bidirectional shunt.
\end{abstract}

\section{An Incidental Finding of Unroofed Coronary Sinus During Angiography Confirmed By 3D Echocardiography; A Case Report}

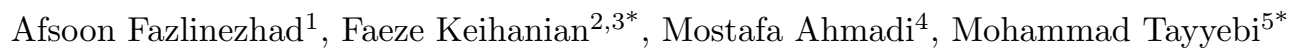

1. M.D., Professor of Cardiology, Fellowship of Echocardiography, Department of Cardiology, Faculty of Medicine, Ghaem Hospital, Mashhad University of Medical Sciences, Mashhad, Iran.

2. M.D. Cardiologist, Cardiology Department, Imam Reza $\mathscr{E}$ Ghaem Hospital, Faculty of Medicine, Mashhad University of Medical Sciences, Mashhad, Iran.

3. M.D. and PhD candidate, Clinician Scientist of Cardiology, Pharmaceutical Research Division, Booali Research Center, Mashhad University of Medical Sciences, Mashhad, Iran

4. M.D., Associated Professor, Interventional cardiologist, Cardiology Department, Faculty of Medicine, Ghaem Hospital, Mashhad University of Medical Sciences, Mashhad, Iran.

5. M.D., Associated Professor, Cardiac Electrophysiology Fellowship, Cardiology Department, Faculty of Medicine, Imam Reza Hospital, Mashhad University of Medical Sciences, Mashhad, Iran.

\section{Abstract}

Unroofed Coronary Sinus (UCS) is an uncommon congenital heart anomaly and the most unusual type of Atrial Septal Defect. This report presents a 71-year-old female with palpitation and dyspnea following an emotional stress, who was referred for ablation of typical atrial flutter. Finally diagnosed with a partially unroofed CS causing a bidirectional shunt.

Keywords: unroofed coronary sinus, atrial septal defect, transesophageal echocardiography, cardiac anomaly

\section{Key Summary Points}

Trans-esophageal echocardiography can be useful in detecting the abnormal anatomy.

Dilated CS, enlarged right heart, and left-to-right shunt flow, particularly without a clearly visualized defect, are clues for UCS. 
* Corresponding Authors: Dr. Mohammad Tayyebi and Dr. Faeze Keihanian, Department of Cardiology, Cardiology Department, Faculty of Medicine, Imam Reza Hospital, Mashhad University of Medical Sciences, Mashhad, Iran. Tel: +989153032232, Email: Tayyebim@mums.ac.ir, Keihanianf941@mums.ac.ir

\section{Abbreviations:}

UCS: Unroofed Coronary Sinus

ASD: Atrial Septum Defect

CCS: Canadian Cardiovascular Society grading

LVEF: Left Ventricular Ejection Fraction

LSVC: Left Superior Vena Cava

RV: Right Ventricle

LA: Left Atrium

RA: Right Atrium

PFO: Patent Foramen Ovale

AI: Aortic Insufficiency

MR: Mitral Regurgitation

TR: Tricuspid Regurgitation

PH: Pulmonary Hypertension

PAP: Pulmonary Arterial Pressure

PVR: Pulmonary Vascular Resistance

TEE: trans-esophageal echocardiography

TTE: trans-thoracic echocardiography

\section{Introduction}

A normal coronary sinus drains the cardiac veins to the right atrium. An Unroofed Coronary Sinus (UCS) drains the cardiac veins and unusually connects with the left atrium. This abnormal communication is because of a deficient in growth of a section between the left atrium and the coronary sinus. An alternative explanation is the subsequent dissolution of this partition (1). The UCS syndrome is a rare congenital heart anomaly (2) and the most uncommon type of atrial septal defect (3), in which there is partial or complete lack of the roof of the coronary sinus (3), and is regularly related to a complex congenital anomaly (4). It is necessary to diagnose this anomaly to determine the patient's prognosis, as it could lead to a brain abscess or cerebral emboli due to right-left shunt. Its diagnosis could be missed because of nonspecific clinical manifestations (5). Here, researchers report a case of UCS with a nonspecific presentation.

\section{Case presentation}

A 71-year-old female was admitted due to the discovery of atrial flutter. She had no known coronary risk factor and no previous history of drug consumption. She was complaining of paroxysmal palpitation, dyspnea (functional class I-II) for the past 3 months, and typical exertional angina in the last week. She had been admitted 3 times due to symptomatic atrial flutter in this period. The laboratory data were normal. On physical examination, she was slightly cyanotic. Cardiac auscultation revealed a loud $\mathrm{P}_{2}$ and wide splitting of $\mathrm{S}_{2}$. The chest X-ray showed cardiomegaly, prominent bilateral pulmonary hilum, and signs of enlarged left atrium. To investigate probable underlying disease causing Right Atrium (RA) dilatation and atrial flutter, echocardiography was requested. TTE was done and showed normal Left Ventricle (LV) size with ejection 
fraction of 55\%; associated with Right Ventricle (RV), RA, and LA dilatation. First, TEE in another center confirmed these data and showed a 2-mm sized PFO with bidirectional shunt. Although there were moderate $\mathrm{TR}$, severe PH $(\mathrm{PAP}=60 \mathrm{mmHg})$, and severely dilated main PA $(4.1 \mathrm{~cm})$, no ASD was found.

In Cath. Lab before ablation, the patient underwent angiography due to her recent chest pain, and showed no obstructive coronary artery disease; however, in the venous phase of the left coronary angiogram, CS was drained into the left atrium before joining the RA in its normal way. Therefore, with the suspicion of UCS, the patient underwent right heart catheterization, and blood gas analysis of the samples from all heart chambers was performed. As seen in Table 1, the results of the blood gas analyses are consistent with bidirectional shunt through the unroofed CS. Repeated TEE with echo expert demonstrated severely dilated coronary sinus $(22 \mathrm{~mm}$ ) with large Thebesian valve. Incomplete UCS in mid portion of the segment (defect size: $14 \mathrm{~mm}$ ) was confirmed by $2 \mathrm{D}, 3 \mathrm{D}$, and contrast study and was associated with significant bidirectional shunt (Figure 1). There was no PLSVC, nor anomalous pulmonary venous connection. Finally, the patient was referred for corrective surgery with the diagnosis of partial UCS (Type III).

\section{Discussion}

The UCS abnormality accounts for less than $1 \%$ of all ASD forms (6). The unroofing of the coronary sinus may be either partial or complete. The UCS may be associated with other congenital heart diseases, including persistent LSVC (5). Accordingly, UCS has been classified morphologically to 4 types by Kirklin and BarrattBoyes, which are as follows (5):

Type I: Completely unroofed with LSVC

Type II: Completely unroofed without LSVC

Type III: Partially unroofed mid-portion

Type IV: Partially unroofed terminal portion.

The diagnosis of UCS has previously been made by cardiac angiography, surgery, or even necropsy (7). Although echocardiography has become one of the main assessment tools, the diagnosis of unroofed coronary sinus remains tricky and needs high degree of suspicion. On the other hand, TEE in obese patients may have a poor echo view. The TEE is largely an invasive procedure that requires premedication and/or sedation in a large number of patients (5). In the current case, the anomaly had been missed in the first TEE, and was suspected in angiography and then confirmed by repeated TEE. One surgical series showed that the condition was correctly diagnosed preoperatively in only 6 out of 11 cases (5). In the current report, the authors presented an incomplete type III UCS with bidirectional shunt and PFO. We doubted the presence of UCS during angiography and confirmed it by performing repeated TEE while the first TEE before angiography failed to diagnose the anomaly. The researchers referred the patient for surgery; however, she refused to undergo an operation.

The UCS could lead to a variety of symptoms. It could appear as asymptomatic, presented with nonspecific complaints, or accompanied by severe dyspnea and symptoms of overt right-sided heart failure from chronic right ventricular volume overload (3). This leads to a diagnosis at an older age or a lack of diagnosis. The clinical presentation of UCS is mainly determined by the size of the defect between the CS and the left atrium (i.e. the degree of left-to-right shunting), and associated anomalies such as a persistent left SVC (i.e. brain abscess or infarction caused by a right-to-left shunt) (8). According to the difficulty of diagnosis by signs or symptoms, UCS should be considered in patients with left-to-right interatrial shunt, unexplained arterial oxygen desaturation, or cerebral complication (4).

TTE is the most commonly used non-invasive examination method, yet propounding portions like CS, which is drained to the posterior wall of the LA or pulmonary veins are not well described by TTE (9). As mentioned earlier, in the current case, the anomalous CS was not reported or even suspected by TTE. However, TEE and cardiac MRI could accurately assess these posterior structures (3). There are now many ways for diagnosing this anomaly better and quicker than previous methods, such as multi-detector CT and 
cardiac MR with their excellent spatial resolution, which allows for the visualization and accurate anatomic and morphological evaluation of the posterior structures of the heart $(3,4)$. Several case reports have suggested a multi-method for detecting this anomaly for better management $(3,7,10)$. Three dimensional TEE could obviously show the posterior cardiac structure and the route from the LA to the CS, before the surgery, as done for the current case (11).

\section{Conclusion}

In conclusion, this case demonstrated the usefulness of TEE in detecting the abnormal anatomy and the pathophysiology associated with the rare congenital anomaly of UCS. This study reported a rare case of type III UCS associated with PFO that presented a non-specific manifestation and compared it with other cases and reviewed the diagnostic approach of UCS.

It is difficult to diagnose UCS because it is rare without specific laboratory findings. Dilated CS, enlarged right heart, and left-to-right shunt flow, particularly without a clearly visualized defect, are clues for UCS. Although CS dilation may occur in other states, UCS should be considered in case of finding an expanding CS. Type I and II UCS could be determined by echocardiography in a patient, who is suspected of ASD; however, for diagnosis of type III and IV, a detailed TEE done by an expert may be needed. In such cases, other cardiac associated anomalies should be considered. Three dimensional TEE could obviously show the posterior structures of the heart and make a figure in a desirable section for analysis in real-time or offline. The authors confirmed that 3D-TEE has practical and clinical applications, as a useful modality complementary to 2D-TEE, for accurate diagnosis of UCS.

\section{Acknowledgement}

Nothing.

\section{Conflict of interest}

None.

\section{Authors' contributions}

MT analyzed and interpreted the patient data regarding the cardiovascular disease and managed patient. $\mathrm{AF}$ helped in data gathering and diagnosis patient in echocardiographic evaluation. MA helped in patient catheterization and management. FK helped in management of patient, diagnosis and was a major contributor in writing the manuscript. All authors read and approved the final manuscript.

\section{Ethical approval}

This study was performed in accordance with the Helsinki declaration. Data published anonymously.

\section{Consent statement}

A written informed consent was obtained from patient.

\section{Data Availability}

Data sharing is not applicable to this article, as no datasets were generated or analyzed during the current study.

\section{Funding}

\section{Nothing}

A written informed consent was obtained from the patient for

publication of identifying data

A written informed consent was obtained from the patient for

publication of identifying data 


\section{Orcid}

Afsoon Fazlinezhad https://orcid.org/0000-0001-9479-2309

Mostafa Ahmadi https://orcid.org/0000-0001-7846-740X

Mohammad Tayyebi https://orcid.org/0000-0003-1551-5595

\section{References}

1. Knauth A, McCarthy KP, Webb S, Ho SY, Allwork SP, Cook AC, et al. Interatrial communication through the mouth of the coronary sinus. Cardiology in the young. 2002;12(04):364-72.

2. Kim H, Choe YH, Park SW, Jun TG, Kang I-S, Yang J-H, et al. Partially unroofed coronary sinus: MDCT and MRI findings. American Journal of Roentgenology. 2010;195(5):W331-W6.

3. Thangaroopan M, Truong QA, Kalra MK, Yared K, Abbara S. Rare case of an unroofed coronary sinus diagnosis by multidetector computed tomography. Circulation. 2009;119(16):e518-e20.

4. Bonardi M, Valentini A, Camporotondo R. Unroofed coronary sinus and persistent left superior vena cava: A case report. Journal of ultrasound. 2012;15(3):179-82.

5. Ootaki Y, Yamaguchi M, Yoshimura N, Oka S, Yoshida M, Hasegawa T. Unroofed coronary sinus syndrome: diagnosis, classification, and surgical treatment. The Journal of thoracic and cardiovascular surgery. $2003 ; 126(5): 1655-6$.

6. Warnes CA, Williams RG, Bashore TM, Child JS, Connolly HM, Dearani JA, et al. ACC/AHA 2008 Guidelines for the Management of Adults With Congenital Heart Disease: Executive Summary: A Report of the American College of Cardiology/American Heart Association Task Force on Practice Guidelines (Writing Committee to Develop Guidelines for the Management of Adults With Congenital Heart Disease) Developed in Collaboration With the American Society of Echocardiography, Heart Rhythm Society, International Society for Adult Congenital Heart Disease, Society for Cardiovascular Angiography and Interventions, and Society of Thoracic Surgeons. Journal of the American College of Cardiology. 2008;52(23):1890-947.

7. Raj V, Joshi S, Ho YC, Kilner PJ. Case report: Completely unroofed coronary sinus with a left superior vena cava draining into the left atrium studied by cardiovascular magnetic resonance. Indian Journal of Radiology and Imaging. 2010;20(3):215.

8. Kim HR, Yoo SM, Lee HY, Rho JY, Yang WI, Moon JY, et al. A Case of Complete Unroofed Coronary Sinus Syndrome Combined With Coronary Sinus Stenosis Leading to Asymptomatic Presentation. Iranian Journal of Radiology. 2015;12(3).

9. Hahm J, Park Y, Lee J, Choi J, Sul J, Lee S, et al. Magnetic resonance imaging of unroofed coronary sinus: three cases. Pediatric cardiology. 2000;21(4):382-7.

10. Aljizeeri A, Dennie CJ, Chan KL, Beauchesne LM. Unroofed coronary sinus atrial septal defect: diagnosis by multimodality cardiac imaging. Echocardiography. 2014;31(7):E228-E9.

11. Yonekura H, Kanazawa S, Miyawaki I, Yamazaki K. Partially unroofed coronary sinus with persistent left superior vena cava: the utility of two and three-dimensional transesophageal echocardiography: a case report. Korean journal of anesthesiology. 2014;67(1):52-6.

Figure Legend

Figure 1- 3D echocardiography of patient; unroofed coronary sinus with defect size of $14 \mathrm{~mm}$ can be seen. 


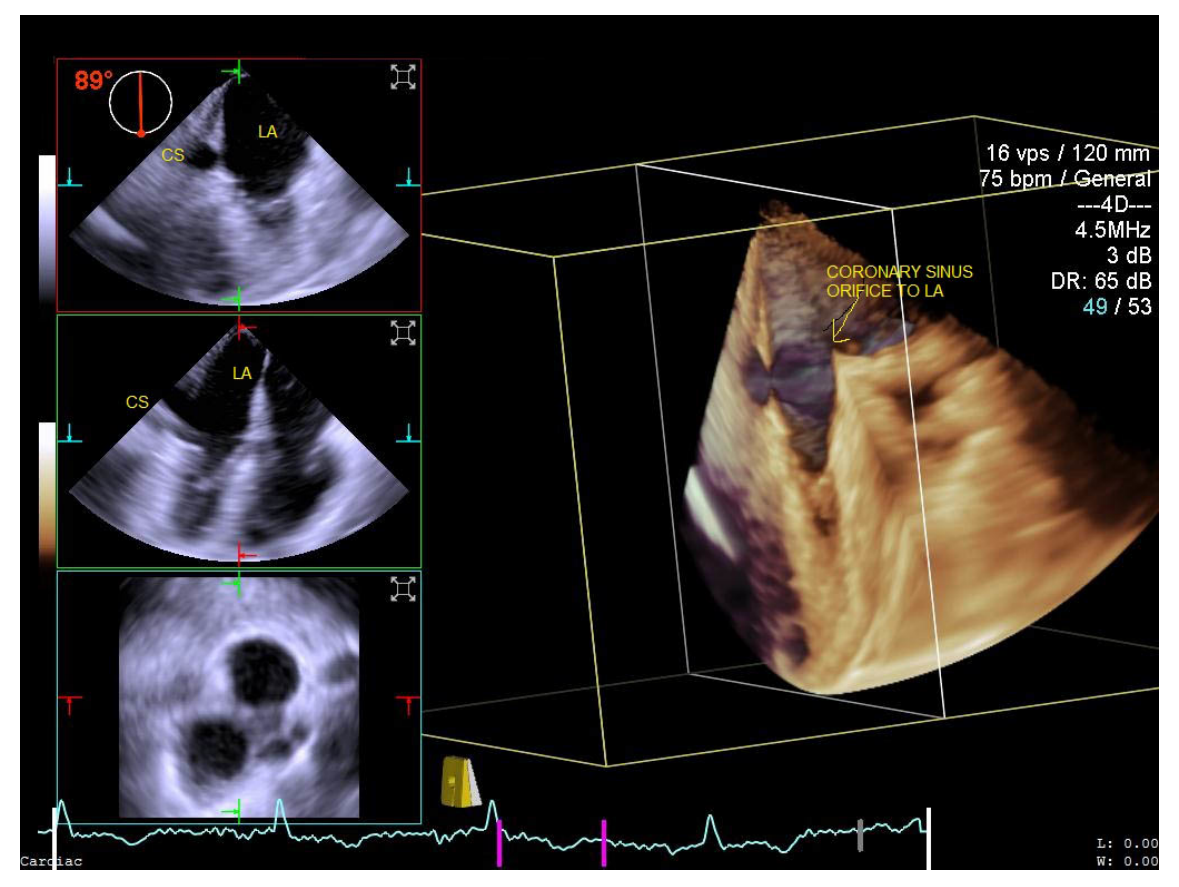

